\title{
Effects of Ginkgo biloba extract and preconditioning on the diabetic rat myocardium
}

\author{
A. Tosaki ${ }^{1}$, T. Pali ${ }^{2}$, M.-T. Droy-Lefaix ${ }^{3}$ \\ ${ }^{1}$ University of Connecticut Health Center, Farmington, Connecticut, USA \\ ${ }^{2}$ Biological Research Center, Szeged, Hungary \\ ${ }^{3}$ IPSEN, Paris, France
}

Summary Effects of preconditioning and Ginkgo biloba extract (EGb 761) were studied in isolated nondiabetic and diabetic ischaemic and re-perfused rat hearts. Hearts were randomly divided into five groups in both the age-matched non-diabetic and the 8-week streptozotocin-induced diabetic groups: Group I, hearts were subjected to $30 \mathrm{~min}$ of global ischaemia followed by 30 min of re-perfusion; Group II, one cycle of preconditioning consisting of $5 \mathrm{~min}$ ischaemia and 10 min re-perfusion before the induction of $30 \mathrm{~min}$ of ischaemia and $30 \mathrm{~min}$ of re-perfusion; Group III, two cycles of preconditioning; Group IV, three cycles; and Group V, four cycles before the onset of $30 \mathrm{~min}$ ischaemia followed by $30 \mathrm{~min}$ of reperfusion. Four cycles of ischaemic preconditioning resulted in a reduction of arrhythmias in non-diabetic rats. Thus, in non-diabetics, the incidence of ventricular fibrillation and tachycardia fell from $92 \%$ and $100 \%$ (no preconditioning) to $33 \%(p<0.05)$ and $42 \%(p<0.05)$, respectively. Four cycles of preconditioning failed to reduce the incidence of re-perfusion arrhythmias in diabetic subjects. Preconditioning reduced the formation of oxygen free radicals measured by electron spin resonance spectroscopy, but the recovery of cardiac function was low in all non-diabetic and diabetic preconditioned groups. EGb 761 at 25 and $50 \mathrm{mg} / \mathrm{kg}$ improved cardiac function in non-preconditioned and preconditioned non-diabetic and diabetic hearts. During re-perfusion in the fourcycle preconditioned non-diabetic and diabetic groups, the amount of free radicals was reduced approximately by 50 and $70 \%$ using 25 and $50 \mathrm{mg} / \mathrm{kg}$ of EGb 761, respectively. EGb 761 improved cardiac function after ischaemia in both non-preconditioned and preconditioned non-diabetic and diabetic rats. Our data suggest that diabetes could abolish the precondition-induced protection. [Diabetologia (1996) 39: 1255-1262]

Keywords Diabetes mellitus, preconditioning, free radicals, Ginkgo biloba extract, arrhythmias.
Repetitive short periods of ischaemia increase the tolerance of the myocardium to a subsequent prolonged ischaemic episode. This cardioprotective effect, which was termed preconditioning, has been shown to occur in various species [1-3] including humans $[4,5]$. In the

Received: 28 March 1996 and in revised form: 3 June 1996

Corresponding author: Dr. M.-T. Droy-Lefaix, IPSEN, 24, Rue Erlanger, F-75781 Paris Cedex 16, France

Abbreviations: EGb 761, Ginkgo biloba extract; ESR, electron spin resonance; DMPO, 5,5-dimethyl-pyrroline-N-oxide; VF, ventricular fibrillation; VT, ventricular tachycardia; HR, heart rate; $\mathrm{CF}$, coronary flow; $\mathrm{AF}$, aortic flow; LVP, left ventricular pressure; $\mathrm{LVdp} / \mathrm{dt}$, first derivative of left ventricular pressure. early 1980s Reimer et al. [3] showed that ATP repletion following a brief period of ischaemia occurred very slowly, raising the possibility that repeated ischaemic periods may lead to cumulative myocardial damage. Surprisingly, brief repeated episodes of ischaemia did not produce any additional ATP depletion, indicating that the rate of ATP breakdown must have been reduced during the extended ischaemic periods [6]. It is possible that a mechanism responsible for slowing the rate of adenine nucleotide depletion may persist and protect the myocardium during a prolonged ischaemic episode [7]. Several mechanisms have been proposed to explain the beneficial effect of preconditioning including reduced accumulation of 
glycolytic metabolites [8]; stimulation of adenosine ${ }_{1}$ receptors [9]; release of prostanoids [10]; activation of protein kinase $\mathrm{C}$ [11]; and a reduction in the rate of ATP utilization in preconditioned hearts [8]. There is a close correlation between myocardial ATP content and the formation of oxygen free radicals [12]; thus, a reduced free radical production may be responsible for the protective effect of preconditioning after an ischaemic/reperfusion period. The preconditioning phenomenon has been extensively studied in hearts obtained from intact (healthy) animals, but only a few studies have been carried out on diabetic hearts [13]. Therefore, we studied whether the preconditioning phenomenon exists in such hearts. The incidence of re-perfusion-induced ventricular fibrillation, (VF), ventricular tachycardia (VT), free radical formation, and cardiac function in isolated hearts from rats with streptozotocin-induced diabetes were recorded. Furthermore, the present study compared the protective efficacy of Ginkgo biloba (EGb 761), a free radical scavenger, and preconditioning in diabetic hearts, and also determined whether the effect might be additive.

Although preconditioning may improve cardiac function during re-perfusion $[14,15]$, some studies have shown that preconditioning did not increase the recovery of heart function during ischaemia/reperfusion [16-18]. In the present study, we used a natural free radical scavenger, extract of Ginkgo biloba (EGb 761, IPSEN, France), to study the recovery of post-ischaemic cardiac function in ischaemic preconditioned diabetic and non-diabetic hearts. EGb 761 is produced from the leaves of the ginkgo tree. After numerous steps of extraction, separation and concentration, the final product is a standardized prescription drug containing $6 \%$ ginkgolides and bilobalide, and $24 \%$ flavonoid Reverosides. We also use electron spin resonance (ESR) spectroscopy to study the effect of preconditioning on free radical formation, because to our knowledge this has not been directly measured previously.

\section{Materials and methods}

Male Sprague-Dawley rats (280-360 g body weight) were used. Animals were anaesthetized with pentobarbital (i. p.), and heparin $(500 \mathrm{IU} / \mathrm{kg})$ was injected i. v. After $20 \mathrm{~s}$ of heparin injection, hearts were excised and placed in perfusion buffer: modified Krebs-Henseleit bicarbonate buffer (in mmol/l: $118 \mathrm{NaCl}, 5.8$ $\mathrm{KCl}, 1.8 \mathrm{CaCl}_{2}, 25 \mathrm{NaHCO}_{3}, 0.36 \mathrm{NaH}_{2} \mathrm{PO}_{4}, 1.2 \mathrm{MgSO}_{4}$ and 5.0 glucose). The isolated working rat heart model was used which has been described in detail elsewhere [19]. The aorta was cannulated and Langendorff perfusion $(100 \mathrm{~cm}$ of water, $10 \mathrm{kPa})$ initiated. During the perfusion the pulmonary vein was cannulated for conversion of the preparation to the working heart mode which was achieved by stopping the Langendorff perfusion and starting left atrial perfusion (at a filling pressure of $17 \mathrm{~cm}$ of the buffer, $1.7 \mathrm{kPa}$ ). Under these conditions the perfusate was ejected spontaneously at a rate of $45-65 \mathrm{ml} / \mathrm{min}$ (measured by a calibrated flow meter) through the aortic cannula against a hydrostatic pressure of $100 \mathrm{~cm}$ of the perfusion buffer. Reduced concentrations of $\mathrm{NaH}_{2} \mathrm{PO}_{4}$ and glucose in the buffer were necessary because these components may disturb the ESR [20]. Coronary effluent from the heart could either be collected and measured or re-circulated. Global ischaemia was imposed by clamping the atrial and aortic cannulas.

All animals were cared for in accordance with the "Principles of Laboratory Animal Care" formulated by the National Society for Medical Research and the "Guide for the Care and Use of Laboratory Animals" prepared by the National Academy of Sciences and published by the National Institute of Health (NIH Publication No. 85-23, revised 1985).

Induction of ischaemia and re-perfusion. After aerobic perfusion of the heart, both the aortic outflow and pulmonary inflow lines were clamped at a point close to the origin of the aortic and pulmonary cannulas; thus, global ischaemia could be maintained for any desired period by clamping the inflow line. Reperfusion could be initiated by unclamping.

Induction of diabetes. Diabetes was induced by an i. v. injection of streptozotocin $(55 \mathrm{mg} / \mathrm{kg})$ dissolved in $0.1 \mathrm{~mol} / \mathrm{l}$ citrate buffer ( $\mathrm{pH} 4.5)$. Non-diabetic age-matched control animals were injected with an equivalent volume of the vehicle (citrate buffer) only. All rats were allowed to drink a $10 \%$ glucose solution for the first $24 \mathrm{~h}$ after the injection of streptozotocin. Diabetes was confirmed by the presence of hyperglycaemia, and insulin levels were measured as described by Vadlamudi and McNeill [21]. In some animals (approximately 12\%), despite the streptozotocin injection, diabetes did not develop. These hearts were excluded from the study and were immediately replaced.

ESR spin-trapping studies. Spin-trapping studies, as we described elsewhere [22-24], were carried out by infusing the spin trap, 5,5-dimethyl-pyrroline-N-oxide (DMPO), through a side arm located just proximal to the end of the heart perfusion cannula. DMPO solution was covered with aluminium foil to prevent light-induced degradation. During re-perfusion in the non-diabetic, diabetic, non-preconditioned, preconditioned, and EGb 761-treated groups, DMPO was directly infused into the heart at a rate of $1 \mathrm{ml} / \mathrm{min}$ of $100 \mathrm{mmol} / \mathrm{l}$ stock solution. This resulted in a final DMPO concentration of $10 \mathrm{mmol} / \mathrm{l}$ because coronary flow was approximately $10 \mathrm{ml} / \mathrm{min}(9.5-$ $11.5 \mathrm{ml} / \mathrm{min}$ ) during Langendorff re-perfusion. This concentration of DMPO did not affect the myocardial contractility and the incidence of arrhythmias [25]. To prevent spin adduct decay, the effluent was immediately frozen as it flowed from the heart with an effluent sampling time of $30 \mathrm{~s}$. The ESR spectra were recorded in a flat quartz cell with a JEOL (JEOL, Tokyo, Japan) JES-PE-1X spectrometer operating at $\mathrm{X}$ band $(9.3$ $\mathrm{MHz}$ ) with a $100-\mathrm{kHz}$ modulation frequency. The microwave power was maintained at $10 \mathrm{~mW}$ to avoid saturation. The scans were traced with 0.2 milli-Tesla of modulation amplitude with 2 min of scan time and with 300 ms of response time. Hyperfine coupling constants were measured directly from the field scan using $\mathrm{Mn}^{2+}$ as a marker for calibration.

Recorded indices. An epicardial ECG was recorded by a polygraph throughout the experimental period with two silver electrodes attached directly to the heart. The ECGs were observed for VF and VT. The heart was considered to be in VF if an irregular undulating baseline was present on the ECG. VT was defined as five or more consecutive premature ventricular complexes, and this classification included repetitive monomorphic VT, which is difficult to dissociate from rapid VT. The heart was considered to be in sinus rhythm if normal sinus 
complexes occurring regularly were present on the ECG. Fibrillating hearts were defribrillated after $3 \mathrm{~min}$ of fibrillation by a defibrillator using two electrodes and $15-\mathrm{V}$ square-wave pulse of $1 \mathrm{~ms}$ duration. Heart rate (HR), coronary flow (CF), aortic flow (AF), left ventricular pressure (LVP), and first derivative of $\mathrm{LVP}(\mathrm{LVdp} / \mathrm{dt})$ were recorded.

Experimental time course. We performed these studies to assess whether one or more episode(s) of $5 \mathrm{~min}$ of normothermic global ischaemia followed by $10 \mathrm{~min}$ of re-perfusion were sufficient to protect the heart from sustained ischaemia followed by re-perfusion, and to estimate the therapeutic value of EGb 761 improving myocardial function in diabetic and non-diabetic preconditioned hearts. Before the isolation of hearts, rats were treated orally with 25 or $50 \mathrm{mg} \cdot \mathrm{kg}^{-1} \cdot$ day $^{-1}$ of EGb 761 for 10 days because this treatment resulted in an effective drug (ginkgoflavon) concentration in the blood [26, 27]. Thus, after 10 days the plasma flavonoid concentrations were $5 \times 10^{3}$ and $6.6 \times 10^{3} \mathrm{mmol} / \mathrm{l}$, respectively. Age-matched control rats received a daily dose of saline solution $(0.9 \% \mathrm{NaCl})$ for 10 days. Hearts were excised and perfused with a drugfree buffer according to the Langendorff method for a 5-min washout period. During the washout period the pulmonary vein was cannulated as described. The studies had two objectives: the first whether the preconditioning (drug-free studies) could reduce the incidence of re-perfusion-induced arrhythmias and improve myocardial function in hearts obtained from diabetic rats, as well as in non-diabetic rats. To achieve this, we used five groups ( $n=12$ in each group) of non-diabetic and diabetic hearts, respectively: (I) hearts were perfused aerobically and subjected to $30 \mathrm{~min}$ ischaemia followed by 30 min of re-perfusion, (II) one cycle of preconditioning consisting of 5-min global ischaemia and 10-min re-perfusion before inducing $30 \mathrm{~min}$ of ischaemia and $30 \mathrm{~min}$ of re-perfusion, (III) two cycles of preconditioning, (IV) three cycles, and (V) four cycles before the onset of $30 \mathrm{~min}$ ischaemia followed by 30 min of re-perfusion. Pre-ischaemic heart function values were registered before the induction of preconditioning in both non-diabetic and diabetic hearts.

The second objective was to study whether EGb 761, an orally administered free radical scavenger, can attenuate the incidence of arrhythmias, and formation of oxygen free radicals, and improve myocardial function in non-diabetic and diabetic preconditioned hearts. The effect of EGb 761 at different dosages $\left(25\right.$ or $\left.50 \mathrm{mg} \cdot \mathrm{kg}^{-1} \cdot \mathrm{day}^{-1}\right)$ was tested in hearts subjected to four cycles of preconditioning before the onset of 30-min ischaemia followed by $30 \mathrm{~min}$ of re-perfusion. Samples were taken at min 3 of re-perfusion (after 30 min of global ischaemia) to measure oxygen free radicals from the effluents of non-diabetic and diabetic hearts. This sampling time was selected according to our previous studies [22, 23].

\section{Statistical analysis}

$\mathrm{HR}, \mathrm{CF}, \mathrm{AF}, \mathrm{LVP}, \mathrm{LVdp} / \mathrm{dt}$, and oxygen free radicals were expressed as mean value \pm SEM. A two-way analysis of variance was first carried out to test for any differences in mean values between groups. If differences were established, the values of the drug-treated groups were compared with those of the drug-free preconditioned non-diabetic and diabetic groups by Dunnett's test. An analogous procedure was followed for distribution of discrete variables, such as the incidence of VF and VT. An overall chi-square test for the $2 \times n$ table was constructed, followed by a sequence of the $2 \times 2$ chi-square test to compare individual groups.
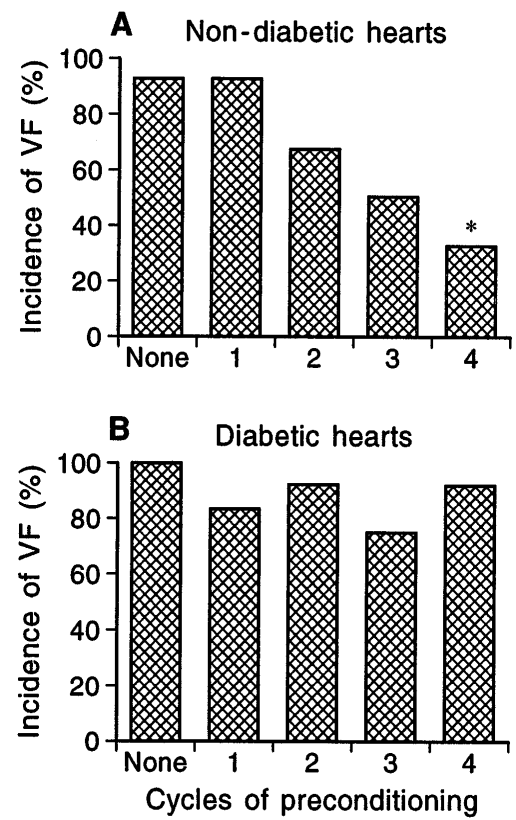

Fig. 1 A, B. Cycles of preconditioning on the incidence (\%) of ventricular fibrillation (VF) during a subsequent sustained 30min ischaemic period followed by re-perfusion in non-diabetic (A) and 8-week-diabetic (B) hearts. No preconditioning (none), one cycle (1), two cycles (2), three cycles (3), four cycles (4). $n=12$ in each group. Incidence of arrhythmias indicates the percentage of 12 hearts showing VF and VT during re-perfusion. ${ }^{*} p<0.05$ compared to no preconditioning

\section{Results}

Arrhythmias. The effect of preconditioning on the incidence of re-perfusion-induced VF is shown in Figure 1. Increasing numbers of preconditioning cycles led to a reduced incidence of VF (Fig. 1A) in non-diabetic hearts, but only four cycles of ischaemia-induced preconditioning resulted in a substantial and significant reduction in the incidence of such arrhythmias. Thus, the incidence of VF (irreversible plus reversible) fell from $92 \%$ in control non-diabetic hearts (no preconditioning) to $33 \%(p<0.05)$ by four cycles of preconditioning. In hearts from 8-weeks-diabetic rats subjected to the preconditioning and ischaemia/ re-perfusion protocol, no such reduction was observed (Fig. 1B) indicating that the preconditioning phenomenon may not occur in diabetic hearts. The incidence of re-perfusion-induced VT followed the same pattern in both non-diabetic and diabetic rats (Fig. 2). These results clearly show that different numbers of precondition cycles, under our experimental conditions, do not reduce the incidence of arrhythmias in diabetic hearts.

In non-preconditioned hearts EGb 761 significantly reduced the incidence of re-perfusion-induced VF in both non-diabetic and diabetic rats (Fig. 3). The drug did not substantially reduce the incidence of VF in non-diabetic preconditioned hearts in comparison with the non-diabetic non-preconditioned 

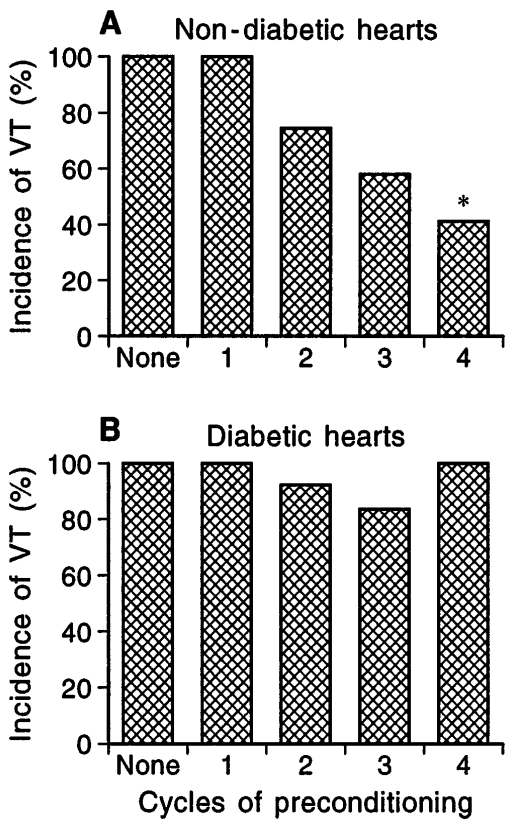

Fig. 2 A, B. Cycles of preconditioning on the incidence (\%) of ventricular tachycardia (VT) during a subsequent sustained 30-min ischaemia followed by re-perfusion in non-diabetic (A) and 8-week-diabetic (B) hearts. No preconditioning (none), one cycle (1), two cycles (2), three cycles (3), four cycles (4). $n=12$ in each group. Incidence of arrhythmias indicates the percentage of 12 hearts showing VF or VT during re-perfusion. $* p<0.05$ compared to no preconditioning
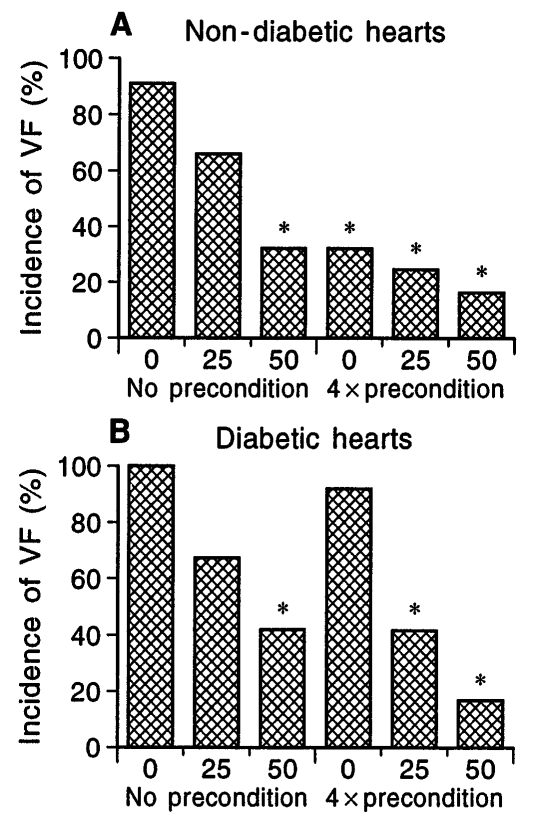

EGb $761(\mathrm{mg} / \mathrm{kg})$

Fig. 3 A, B. Effect of EGb 761 on incidence (\%) of re-perfusion-induced ventricular fibrillation (VF) in non-preconditioned and four-cycle $(4 \times$ precondition) preconditioned ischaemic/re-perfused non-diabetic (A) and 8-week-diabetic (B) hearts; $n=12$ in each group. Incidence of VF shows in how many of 12 hearts VF occurred during re-perfusion. Comparisons were made to the control (EGb 761-free) non-diabetic non-preconditioned (A) and diabetic non-preconditioned (B) groups, respectively $(* p<0.05)$
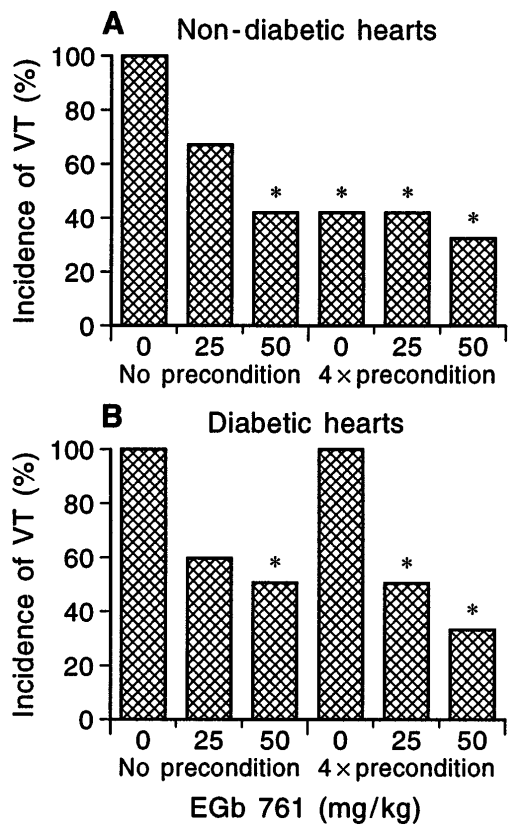

Fig. 4 A, B. Effect of EGb 761 on incidence (\%) of re-perfusion-induced ventricular tachycardia (VT) in non-preconditioned and four-cycle $(4 \times$ precondition $)$ preconditioned ischaemic/re-perfused non-diabetic (A) and 8-week-diabetic (B) hearts; $n=12$ each group. Incidence of VT shows in how many of 12 hearts VT occurred during re-perfusion. Comparisons were made to the control (EGb 761-free) non-diabetic non-preconditioned (A) and diabetic non-preconditioned drug-free $(\mathbf{B})$ groups, respectively $(* p<0.05)$

drug-treated groups (Fig. 3A). In the 8-week-diabetic groups, four cycles of precondition alone failed to reduce the incidence of VF, but 25 and $50 \mathrm{mg} / \mathrm{kg} \mathrm{EGb}$ 761 substantially reduced the incidence of such arrhythmias (Fig. 3B). Thus, the incidence of VF (Fig. 3B) was significantly reduced $(p<0.05)$ from the 8-week-diabetic drug-free value (four-cycle precondition) of $92 \%$ to $41 \%$ and $17 \%$ with 25 and $50 \mathrm{mg} / \mathrm{kg}$ of EGb 761, respectively. With $50 \mathrm{mg} / \mathrm{kg}$ EGb 761, the incidence of re-perfusion-induced VT (Fig.4) was reduced in both non-preconditioned non-diabetic and diabetic hearts, but no such reduction in diabetic preconditioned hearts treated with EGb 761 (Fig. 4B) in comparison with the diabetic non-preconditioned drug-treated value was observed. The incidence of VT was significantly reduced with the doses of 25 and $50 \mathrm{mg} / \mathrm{kg}$ of EGb 761 from their diabetic drug-free preconditioned value of $100 \%$ (Fig. $4 \mathrm{~B})$ to $50 \%(p<0.05)$ and $33 \%$ $(p<0.05)$, respectively. The protection afforded by EGb 761 against the incidence of arrhythmias was unrelated to the plasma glucose levels, since there was no significant change between plasma glucose measured in diabetic drug-free and diabetic drug-treated groups (Table 1). In the 8-week-diabetic group, serum insulin was reduced from its control age-matched non-diabetic value of $354 \pm 32$ to $149 \pm 18$ $\mathrm{pmol} / \mathrm{l}(p<0.05)$. This approximate $60 \%$ reduction 
Table 1. Serum glucose (mmol/l) in non-diabetic age-matched control and 8-week-diabetic rats

\begin{tabular}{llllll}
\hline Groups & \multicolumn{4}{l}{ Cycles of preconditioning } \\
\cline { 2 - 5 } & None & 1 & 2 & 3 \\
\hline Non-diabetic (age-matched) & $7 \pm 0.4$ & $7.3 \pm 0.4$ & $7.6 \pm 0.5$ & $6.7 \pm 0.4$ \\
8-week diabetic & $27 \pm 0.7$ & $29 \pm 1.1$ & $27 \pm 0.6$ & $27 \pm 0.8$ & $7.4 \pm 0.5$ \\
8-week diabetic (+25 mg/kg EGb) & NR & NR & NR & NR & $28 \pm 0.72$ \\
8-week diabetic (+50 mg/kg EGb) & NR & NR & NR & $29 \pm 0.8$ \\
\hline
\end{tabular}

Data are mean \pm SEM. $n=12$ in each group. Comparisons were made between the 8 -week diabetic drug-free values and the drugtreated values

NR, Not recorded

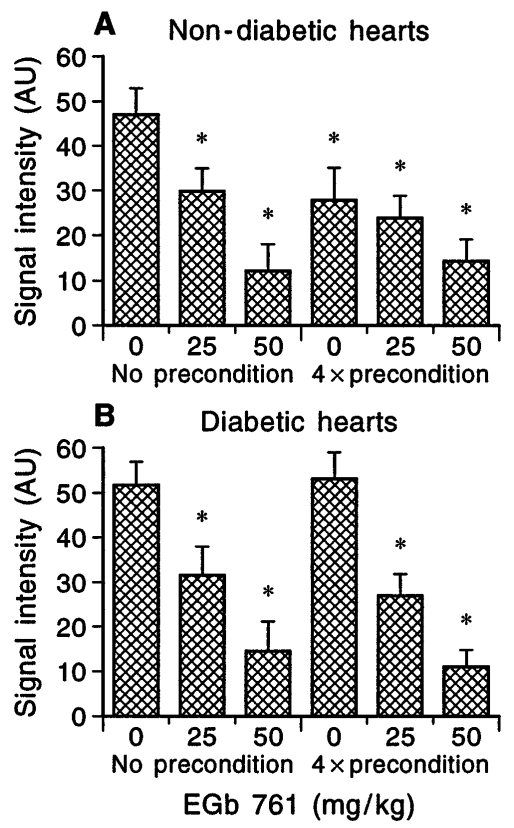

Fig. 5 A, B. Four cycles of preconditioning on the signal intensity (arbitrary units AU) of DMPO-OH adduct (free radical adduct) during a subsequent 30 -min ischaemia followed by 3 min of re-perfusion, with and without EGb 761 treatment, in non-diabetic $(\mathbf{A})$ and diabetic $(\mathbf{B})$ hearts. Four cycles of preconditioning ( $4 \times$ precondition); $n=12$ each group. Comparisons were made to the non-preconditioned non-diabetic drugfree (A) and non-preconditioned diabetic (B) EGb 761-free groups, respectively $(* p<0.05)$

was the same in all EGb 761-treated animals, and the drug failed to increase serum insulin levels in diabetic rats.

ESR studies. We previously reported $[22,23]$ that after $1 \mathrm{~min}$ of re-perfusion oxygen free radical formation was observed, consisting of 1:2:2:1 signal quartet. On examination of the time course for the appearance of the DMPO-OH signal, maximum signal intensity was observed at $3 \mathrm{~min}$ of re-perfusion, therefore this time point was selected to measure oxygen free radical formation in the preconditioned drug-free and drug-treated non-diabetic and diabetic groups. Four cycles of preconditioning reduced the formation of oxygen free radicals by approximately $50 \%$ (Fig. 5A) in the non-diabetic group compared to the non-diabetic non-preconditioned group. The results (Fig. 5A) show that 25 and $50 \mathrm{mg} / \mathrm{kg}$ of EGb 761 resulted in a further reduction in the DMPO$\mathrm{OH}$ signal intensity indicating a reduced free radical formation in the non-diabetic preconditioned groups. This reduction was reflected in a striking reduction in the incidence of arrhythmias (Figs. 3A and 4A). In diabetic hearts (Fig. 5B), four cycles of precondition failed to reduce the formation of free radicals, indicating the lack of precondition-induced protection on their release. EGb 761 ( 25 and $50 \mathrm{mg} / \mathrm{kg}$ ) significantly reduced the signal intensity of oxygen radical formation both in preconditioned diabetic and nonpreconditioned diabetic hearts, showing the pathological importance of free radicals in diabetic, as well as in non-diabetic rats (Fig. 5B). These results also show that four cycles of preconditioning (Fig. 5B) did not reduce the formation of free radicals in the drug-free diabetic hearts $(54 \pm 6$ arbitrary units) compared to the non-preconditioned diabetic hearts (53 \pm 4 arbitrary units), the reduction observed in free radical production in diabetic hearts clearly depending on the presence of EGb 761. In the non-preconditioned diabetic group (Fig.5B), EGb 761 itself reduced the formation of free radicals, as well as in the four-cycle preconditioned diabetic group.

Pre- and post-ischaemic cardiac function. Table 2 shows the absolute values for pre-ischaemic myocardial function in the drug-free age-matched control, diabetic, and drug-treated non-diabetic and diabetic groups. After 8 weeks of diabetes, a significant reduction was observed in AF, LVP, and LVdp/dt compared the age-matched non-diabetic values, indicating the development of diabetes-induced cardiac failure (Table 2). With $50 \mathrm{mg} / \mathrm{kg}$ of EGb 761, a significant improvement in pre-ischaemic cardiac function (AF, LVP, and LVdp/dt) was observed compared to the drug-free 8-week-diabetic group.

Four cycles of preconditioning followed by $30 \mathrm{~min}$ of ischaemia and $30 \mathrm{~min}$ of re-perfusion failed to improve the recovery of cardiac function in both non-diabetic and diabetic hearts. EGb $761(50 \mathrm{mg} / \mathrm{kg}) \mathrm{im}-$ proved the post-ischaemic myocardial function in the four-cycle preconditioned non-diabetic and 
Table 2. Cardiac function before preconditioning and the induction of ischaemia (pre-ischaemic values)

\begin{tabular}{|c|c|c|c|c|c|}
\hline \multirow[t]{2}{*}{ Groups } & \multicolumn{5}{|l|}{ Cardiac function } \\
\hline & HR (beats/min) & $\mathrm{CF}(\mathrm{ml} / \mathrm{min})$ & $\mathrm{AF}(\mathrm{ml} / \mathrm{min})$ & $\mathrm{LVP}(\mathrm{kPa})$ & $\mathrm{LV} \mathrm{dp} / \mathrm{dt}(\mathrm{kPa} / \mathrm{s})$ \\
\hline Non-diabetic, age-matched control & $320 \pm 7$ & $27.6 \pm 0.9$ & $50.6 \pm 1.6$ & $17.4 \pm 0.3$ & $782 \pm 24$ \\
\hline $\begin{array}{l}\text { Non-diabetic, age-matched control } \\
(50 \mathrm{mg} / \mathrm{kg} \mathrm{EGb})\end{array}$ & $323 \pm 6$ & $27.2 \pm 0.8$ & $51.1 \pm 2.0$ & $16.9 \pm 0.5$ & $790 \pm 28$ \\
\hline 8-week diabetic ( $25 \mathrm{mg} / \mathrm{kg} \mathrm{EGb)}$ & $308 \pm 9$ & $28.2 \pm 1.2$ & $42.3 \pm 2.0^{\mathrm{a}}$ & $15.5 \pm 0.5^{\mathrm{a}}$ & $679 \pm 35^{\mathrm{a}}$ \\
\hline 8-week diabetic (50 mg/kg EGb) & $311 \pm 8$ & $26.6 \pm 1.3$ & $48.0 \pm 1.9^{\mathrm{b}}$ & $17.0 \pm 0.4^{\mathrm{b}}$ & $748 \pm 28^{\mathrm{b}}$ \\
\hline
\end{tabular}

Values are mean \pm SEM

$n=12$ in each group

${ }^{a}$ Compared to the nondiabetic age-matched control group $(p<0.05)$

${ }^{\mathrm{b}}$ Compared to the 8 -week diabetic values $(p<0.05)$

Table 3. Cardiac function during re-perfusion (after preconditioning and ischaemia)

\begin{tabular}{|c|c|c|c|c|c|}
\hline \multirow[t]{2}{*}{ Groups } & \multicolumn{5}{|l|}{ Cardiac function } \\
\hline & HR (beats/min) & $\mathrm{CF}(\mathrm{ml} / \mathrm{min})$ & $\mathrm{AF}(\mathrm{ml} / \mathrm{min})$ & LVP (kPa) & $\mathrm{LV}$ dp/dt $(\mathrm{kPa} / \mathrm{s})$ \\
\hline Non-diabetic, age-matched control & $293 \pm 7$ & $17.2 \pm 0.5$ & $11.4 \pm 1.2$ & $10.8 \pm 0.4$ & $448 \pm 24$ \\
\hline $\begin{array}{l}\text { Non-diabetic, age-matched control } \\
(+4 \times \mathrm{PC}+25 \mathrm{mg} / \mathrm{kg} \mathrm{EGb})\end{array}$ & $289 \pm 8$ & $18.1 \pm 0.6$ & $12.1 \pm 0.8$ & $11.6 \pm 0.5$ & $468 \pm 31$ \\
\hline 8-week diabetic & $285 \pm 9$ & $16.9 \pm 0.6$ & $4.0 \pm 0.5^{\mathrm{a}}$ & $5.9 \pm 0.4^{\mathrm{a}}$ & $309 \pm 17^{\mathrm{a}}$ \\
\hline 8 -week diabetic $(+4 \times \mathrm{PC})$ & $280 \pm 8$ & $17.9 \pm 0.6$ & $4.8 \pm 0.6^{\mathrm{a}}$ & $6.3 \pm 0.5^{\mathrm{a}}$ & $330 \pm 20^{\mathrm{a}}$ \\
\hline $\begin{array}{l}\text { 8-week diabetic } \\
(+4 \times \mathrm{PC} \text { and } 25 \mathrm{mg} / \mathrm{kg} \mathrm{EGb})\end{array}$ & $287 \pm 7$ & $18.1 \pm 0.7$ & $7.8 \pm 0.6^{\mathrm{a}, \mathrm{b}}$ & $8.4 \pm 0.4^{a, b}$ & $385 \pm 21^{\mathrm{a}, \mathrm{b}}$ \\
\hline $\begin{array}{l}\text { 8-week diabetic } \\
(+4 \times \mathrm{PC} \text { and } 50 \mathrm{mg} / \mathrm{kg} \mathrm{EGb})\end{array}$ & $302 \pm 9$ & $18.0 \pm 0.5$ & $10.8 \pm 0.8^{\mathrm{b}}$ & $10.2 \pm 0.5^{\mathrm{b}}$ & $420 \pm 19^{b}$ \\
\hline
\end{tabular}

Mean \pm SEM. $n=12$ in each group.

${ }^{a}$ Compared to non-diabetic age-matched control group.

${ }^{\mathrm{b}}$ Compared to 8-week diabetic values $p<0.05$

diabetic groups compared to the four-cycle preconditioned non-diabetic and diabetic drug-free group, respectively (Table 3 ).

\section{Discussion}

The precondition-mediated protection of cardiac function may be of interest in the clinical situation since recurrent episodes of myocardial ischaemia are commonly observed in patients with coronary artery disease who suffer from frequent angina pectoris or who have undergone angioplasty of the left anterior descending coronary artery [4, 28, 29].

We have demonstrated that increasing cycles of ischaemic preconditioning can reduce the incidence of arrhythmias in the myocardium after a prolonged period of normothermic global ischaemia in non-diabetic intact (healthy) hearts. We have also shown that hearts from 8-week-diabetic rats may not be preconditioned, indicating that diabetes could abolish the precondition-mediated cardiac protection. In addition, we studied the effects of EGb 761 (a free radical scavenger), a ginkgoflavon-rich extract [30] to determine the importance of free radicals on the recovery of myocardial function in preconditioned non-diabetic and diabetic hearts. In the drug-free four-cycle preconditioned non-diabetic group, recovery of AF, $\mathrm{LVP}$, and $\mathrm{LVdp} / \mathrm{dt}_{\max }$ was significantly lower compared to the drug-treated non-diabetic preconditioned groups.

In the diabetic group, preconditioning alone failed to reduce the incidence of re-perfusion-induced arrhythmias and improve the recovery of post-ischaemic cardiac function indicating a lack of preconditioning-induced protection in diabetic hearts. Administration of EGb 761 resulted in a significant reduction in incidence of re-perfusion-induced arrhythmias, and 
improvement in cardiac contractility in preconditioned diabetic as well as non-preconditioned diabetic rats. This was reflected in a reduction of free radical production measured by ESR spectroscopy indicating that EGb 761, not the preconditioning, was responsible for free radical reduction in the diabetic hearts. Our findings, in agreement with others [24], emphasize the pathological importance of free radicals in ischaemic/re-perfused hearts, and that the preconditioning phenomenon itself, in comparison with the non-diabetic intact hearts, does not play a major role in ischaemia/re-perfusion-induced injury in human insulin-dependent diabetic subjects. Some studies also show a lack of precondition-induced protection in the improvement of myocardial function during re-perfusion $[16,18]$ in intact myocardium.

Flavonoids have been shown to modulate P-450dependent metabolic activities in vitro and in vivo [31], and to scavenge $\cdot \mathrm{O}_{2}^{-}$and $\cdot \mathrm{OH}$ free radicals in alpha-radiolysis studies [32]. They also act as antioxidants [33] and have anti-inflammatory actions [34]. The specific action of flavonoids, as well as EGb 761, may be to increase the binding affinity of a substrate [35] or to improve the electron transfer efficacy between NADPH-cytochrome P-450 reductase and the P-450 enzyme.

Preconditioning can preserve myocardial ATP content, and as reported by Murry et al. [7] ischaemic preconditioning is characterized by decreased ATP catabolism and reduced glycolysis. Because ATP breakdown during ischaemia plays an important role in the free radical production during re-perfusion, a reduced production may be responsible for the protective effect of preconditioning. Although free radical generation and oxidant stress appear to be important in the genesis of re-perfusion-induced arrhythmias $[24,36]$ there is little evidence that they play an important role in preconditioning. Our previous study shows that free radical pathways are involved in the mechanism(s) of preconditioning in non-diabetic intact hearts [37]. Furthermore, precondition itself does not afford functional cardiac protection in diabetic hearts in our model, and the free radical pathways may play a more important role than the preconditioning-induced protection (via other mechanisms) in diabetic subjects. It is not our intention to suggest here that only free radical-induced arrhythmias exist, nor to suggest that they operate alone or over tightly constrained time spans. It seems likely that many overlapping triggers exist such as prostaglandins, free radicals, adenosine $\mathrm{A}_{1}$ receptors, protein kinase $\mathrm{C}$, ATP-sensitive $\mathrm{K}$ channels, and maldistribution of ions particularly $\mathrm{Na}, \mathrm{K}$, and $\mathrm{Ca}[24,38]$.

Many precondition studies have been performed in hearts obtained from intact (healthy) animals, but few results are available in preconditioned diseased (e.g. diabetic) myocardium. Thus, Liu et al. [13] found that preconditioning could reduce infarct size in type 2 diabetic rats. It is not clear, from their studies, whether the changes in cellular metabolism resulting in protection in type 2 diabetic rats were amplified by superimposed preconditioning or whether the latter causes a further effect by an unrelated mechanism. Our results suggest that the precondition has no beneficial effect on ischaemic/re-perfused myocardium obtained from type 1 diabetic rats. A wide range of biochemical changes have been described in the ventricular muscle of diabetic rats, and can be classified as: (i) alterations in myosin ATPase and myosin isoenzyme distribution [39], (ii) changes in $\mathrm{Ca}^{2+}$ transport [40], (iii) alterations in adrenergic and cholinergic receptors [41], and (iv) changes in carbohydrate, lipid, and adenine nucleotide metabolism [42]. All or any of these biochemical changes in the diabetic myocardium may be responsible for the lack of the protective effect of preconditioning in insulin-dependent diabetic myocardium. These findings indicate that the mechanisms responsible for the arrhythmogenesis are different in diabetic and nondiabetic subjects, and diabetes could abolish the precondition-induced cardiac protection.

Although preconditioning "cycle-dependently" reduces the incidence of arrhythmias in non-diabetic hearts, it did not attenuate myocardial dysfunction [18] during re-perfusion (depressed myocardial function). The administration of a free radical scavenger, EGb 761, significantly reduced the incidence of arrhythmias and improved myocardial contractility in both non-diabetic and diabetic preconditioned hearts. If preconditioning itself improved recovery of cardiac function, data points for the preconditioned drug-free groups would be expected to lie above the points for the control and EGb 761-treated groups. Our results show that preconditioning alone is not sufficient to substantially improve myocardial contractility. However, our studies show an absence of additional deterioration in myocardial function or injury after prolonged myocardial ischaemia in the presence of previous ischaemic events. Therefore, it is safe to assume that brief ischaemic episodes do not cause additive tissue injury when isolated rat hearts have been subjected to subsequent prolonged periods of ischaemia and re-perfusion.

\section{References}

1. Ferdinandy P, Szilvassy Z, Csont T et al. (1995) Nitroglycerininduced direct protection of the ischaemic myocardium in isolated working hearts of rats with vascular tolerance to nitroglycerin. Br J Pharmacol 115: 1129-1131

2. Szilvassy Z, Ferdinandy P, Bor P, Jakab I, Lonovics J, Koltai M (1994) Ventricular overdrive pacing-induced anti-ischaemic effect: a conscious rabbit model of preconditioning. Am J Physiol 266:H2033-H2041

3. Reimer KA, Hill ML, Jennings RB (1981) Prolonged depletion of ATP and the adenine nucleotide pool due to delayed 
resynthesis of adenine nucleotides following reversible myocardial ischaemic injury in dogs. J Mol Cell Cardiol 13: 229-239

4. Deutsch E, Berger M, Kussmaul WG, Hirshfeld JW, Herrmann HC, Laskey WK (1990) Adaptation to ischaemia during percutaneous transluminal coronary angioplasty: clinical, hemodynamic, and metabolic features. Circulation 82: 2044-2051

5. Yellon DM, Alkhulaifi AM, Pugsley WB (1993) Preconditioning the human myocardium. Lancet 342: 276-277

6. Reimer KA, Murry CE, Yamasawa I, Hill ML, Jennings RB (1986) Four brief periods of myocardial ischaemia cause no cumulative ATP loss or necrosis. Am J Physiol 251:H1306-H1315

7. Murry CE, Jennings RB, Reimer KA (1986) Preconditioning with ischaemia: a delay of lethal cell injury in ischaemic myocardium. Circulation 74: 1124-1136

8. Murry CE, Richard VJ, Reimer KA, Jennings RB (1990) Ischaemic preconditioning slows energy metabolism and delays ultrastructural damage during a sustained ischaemic episode. Circ Res 66: 913-931

9. Liu GS, Thornton J, Van Winkle DM, Stanley AWH, Olsson RA, Downey JM (1991) Protection against infarction afforded by preconditioning is mediated by $\mathrm{A}_{1}$ receptors in rabbit heart. Am J Physiol 84:H350-H356

10. Vegh A, Szekeres L, Parratt JR (1990) Protective effects of preconditioning of the ischaemic myocardium involve cyclooxygenase products. Cardiovasc Res 24: 1020-1023

11. Zhou X, Zhai X, Ashraf M (1996) Preconditioning of bovine endothelial cells: the protective effect is mediated by an adenosine $\mathrm{A}_{2}$ receptor through a protein kinase $\mathrm{C}$ signaling pathway. Circ Res 78: 73-81

12. Goldhaber JI, Lamp ST, Weiss JN (1989) Effects of exogenous free radicals on electromechanical function and metabolism in isolated rabbit and guinea pig ventricle. J Clin Invest 83: 1800-1809

13. Liu Y, Thornton JD, Cohen MV, Downey JM, Schaffer SW (1993) Streptozotocin-induced non-insulin-dependent diabetes protects the heart from infarction. Circulation 88: 1273-1278

14. Asimakis GK, Inners-McBride K, Conti VR (1993) Attenuation of postischaemic dysfunction by ischaemic preconditioning is not mediated by adenosine in the isolated rat heart. Cardiovasc Res 27: 1522-1530

15. Lasley RD, Anderson GM, Mentzer RM (1993) Ischaemic and hypoxic preconditioning enhance postischaemic recovery of function in the rat heart. Cardiovasc Res 27: 565-570

16. Sandhu R, Diaz RJ, Wilson GJ (1993) Comparison of ischaemic preconditioning in blood perfused and buffer perfused isolated heart models. Cardiovasc Res 27: 602-607

17. Tosaki A, Cordis GA, Szerdahelyi P, Engelman RM, Das DK (1994) Effects of preconditioning on re-perfusion arrhythmias, myocardial functions, formation of free radicals, and ion shifts in isolated ischaemic/re-perfused rat hearts. J Cardiovasc Pharmacol 23: 365-373

18. Yang BC, Nicolini FA, Nichols WW, Mehta JL (1994) Failure of brief ischaemic episodes to protect against myocardial dysfunction caused by ischaemia and re-perfusion in isolated rat hearts. Am Heart J 128: 1192-1200

19. Tosaki A, Hellegouarch A (1994) Adenosine triphosphate-sensitive potassium channel blocking agent ameliorates, but the opening agent aggravates, ischaemia/re-perfusion-induced injury: heart function studies in nonfibrillating isolated hearts. J Am Coll Cardiol 23: 487-496

20. Blasig IE, Ebert B, Hennig C, Pali T, Tosaki A (1990) Inverse relationship between ESR spin trapping of oxyradicals and degree of functional recovery during myocardial re-perfusion in isolated working rat heart. Cardiovasc Res 24: 263-270

21. Vadlamudi RVSV, McNeill JH (1983) Effect of alloxan and streptozotocin-induced diabetes on isolated rat heart responsiveness to carbachol. J Pharmacol Exp Ther 225: 410-415

22. Tosaki A, Blasig IE, Pali T, Ebert B (1990) Heart protection and radical trapping by DMPO during re-perfusion in isolated working rat hearts. Free Rad Biol Med 8: 363-372
23. Tosaki A, Braquet P (1990) DMPO and re-perfusion injury: arrhythmia, heart function, electron spin resonance, and nuclear magnetic resonance studies in isolated working guinea pig hearts. Am Heart J 120: 819-830

24. Ambrosio G, Zweier JL, Jacobus WE, Weisfeldt ML, Flaherty JT (1987) Improvement of postischaemic myocardial function and metabolism induced by administration of desferrioxamine at the time of reflow: the role of iron in the pathogenesis of re-perfusion injury. Circulation 76: 906-915

25. Hearse DJ, Tosaki A (1987) Re-perfusion-induced arrhythmias and free radicals: studies in the rat heart with DMPO. J Cardiovasc Pharmacol 9: 641-650

26. Szabo ME, Droy-Lefaix MT, Doly M, Carre C, Braquet P (1991) Ischaemia and re-perfusion-induced histologic changes in rat retina: demonstration of a free radical-mediated mechanism. Invest Ophthalmol Vis Sci 32: 1471-1478

27. Drieu K (1986) Preparation et definition de l'extrait de Ginkgo biloba. La Presse Medicale 31: 1455-1457

28. Cribier A, Korsatz L, Koning R et al. (1992) Improved myocardial ischaemic response and enhanced collateral circulation with long repetitive coronary occlusion during angioplasty: a prospective study. J Am Coll Cardiol 20: 578-586

29. Kloner RA, Shook T, Przyklenk K et al. (1995) Previous angina alters in hospital outcome in TIMI 4: a clinical correlate to preconditioning? Circulation 91: 37-47

30. Marcocci L, Packer L, Droy-Lefaix MT, Sekaki A, GardesAlbert M (1994) Antioxidant action of Ginkgo biloba extract EGb 761. Methods Enzymol 234: 462-475

31. Lasker JM, Huang MT, Conney AH (1982) In vivo activation of zoxazolamine metabolism by flavone. Science 216 : 1419-1421

32. Gardes-Albert M, Sekaki A, Ferradini C, Droy-Lefaix MT (1990) EGb 761 scavenger effect against $\cdot \mathrm{OH}$ and $\cdot \mathrm{O}_{2}^{-}$free radicals: an alpha-radiolysis study. Free Rad Biol Med 9: 190 (Abstract)

33. Slater TF, Cheeseman KH, Davies MJ (1987) Free radical scavenging properties of modulators of eicosanoid metabolism. Adv Prostaglandin Thromboxane Leuko Res 17: 1098-1102

34. Gabor M (1979) Anti-inflammatory substances of plant origin. In: Vane JR, Ferreira SH (eds) Handbook of experimental pharmacology. Vol. 50, part 2: Anti-inflammatory drugs. Springer, Berlin pp 698-739

35. Johnson EF, Schwab GE, Vickery LE (1988) Positive effectors of the binding of an active site-directed amino steroid to rabbit cytochrome P-450 3c. J Biol Chem 263: 17672-17677

36. Hearse DJ, Tosaki A (1988) Free radicals and calcium: simultaneous interacting triggers as determinants of vulnerability to re-perfusion-induced arrhythmias in the rat heart. J Mol Cell Cardiol 20: 213-223

37. Tosaki A, Engelman DT, Pali T, Engelman RM, Droy-Lefaix MT (1994) Ginkgo biloba extract (EGb 761) improves postischaemic function in isolated preconditioned working rat hearts. Cor Artery Dis 5: 443-450

38. Hu K, Nattel S (1995) Mechanisms of ischaemic preconditioning in rat hearts: involvement of $\alpha_{1 \mathrm{~B}}$-adrenoceptors, pertussis toxin-sensitive $\mathrm{G}$ proteins, and protein kinase $\mathrm{C}$. Circulation 92: 2259-2265

39. Garber DW, Everett AW, Neely JR (1983) Cardiac function and myosin ATPase in diabetic rats treated with insulin, T3, and T4. Am J Physiol 244:H592-H598

40. Heyliger CE, Prakash A, McNeill JH (1987) Alterations in cardiac sarcolemmal $\mathrm{Ca}^{2+}$ pump activity during diabetes mellitus. Am J Physiol 252:H540-H544

41. Xiang H, McNeill JH (1991) Alpha 1-adrenoceptor-mediated phosphoinositide breakdown and inotropic responses in diabetic hearts. Am J Physiol 260:H557-H562

42. Rodrigues B, McNeill JH (1992) The diabetic heart: metabolic cause for the development of a cardiomyopathy. Cardiovasc Res 26: 913-922 